\title{
Analysis of a double steering forest trailer for long wood log transportation
}

\author{
Francesco Marinello, Stefano Grigolato, Luigi Sartori, Raffaele Cavalli \\ Department of Land, Environment, Agriculture and Forestry, University of Padova, Legnaro (PD), \\ Italy
}

\begin{abstract}
A cost effective technical solution of a forest double steering trailer was studied and tested, allowing decrease of radius of curvature and increase of the maneuverability, independently from the length of the transported logs. The steering system improves the so called "stingertype truck" configuration using an articulated frame; through a rearward hinge, the front wheels direction mechanically controls and adapts the direction of rear twin wheels. The study was based on CAD simulations considering the dimensions of the trailer transporting logs with a length up to $12 \mathrm{~m}$. For different log lengths the simulation analyzed the total maneuver area, in terms of minimum curve radius and curve widening. A field test on a prototype operated for transport of long logs along a representative forest road in the Alps was carried out. The results confirm the improvements foreseen by CAD simulations, with allowed curve radius down to $6 \mathrm{~m}$, even with $12 \mathrm{~m}$ logs.
\end{abstract}

\section{Introduction}

The transportation of wood both on-road and off-road represents the backbone of harvesting operations over gentle as well as steep terrain (Bont et al. 2012).

In mountainous area on-road transport is often operated on steep terrains in which the construction and maintenance of the forest roads determine high costs (Stückelberger, et al. 2006). Therefore the forest road networks in mountainous area consist of narrow and single lanes, high road gradients and frequent switchback curves (Cavalli and Grigolato, 2010).

As a consequence road standard characteristics strongly influence the transportation feasibility by truck and trailer system in mountainous area. As a result tractor and forest trailer systems, commonly used for off-road forwarding of logs along the skidding trails network, in the

Correspondence: Raffaele Cavalli, Department of Land, Environment, Agriculture and Forestry - University of Padova, Legnaro (PD), Italy E-mail: raffaele.cavalli@unipd.it.

Key words: forest double steering trailer, CAD simulations, forest road, wood transport.

(C) Copyright F. Marinello et al., 2013

Licensee PAGEPress, Italy

Journal of Agricultural Engineering 2013; XLIV(s2):e3

doi:10.4081/jae.2013.s2.e3

This article is distributed under the terms of the Creative Commons Attribution Noncommercial License (by-nc 3.0) which permits any noncommercial use, distribution, and reproduction in any medium, provided the original author(s) and source are credited. case of extreme conditions represent the only suitable transportation system in such severe conditions (Baldini et al. 2010).

A wide range of logging trailers is available. Commonly they are designed in order to minimizing the construction weight and maximizing the payload. The chassis spine (single or double), where the wheels' cross-members and the stake's bolsters are attached, is the fundamental part of the trailer's frame (Jones, 1995).

According to technical reports of forest trailers companies, most popular tractor and trailer systems have payloads ranging from 5 to 10 tons. Lower capacity trailers may have only two wheels but usually most forest models have a four wheel bogie design. Also steerable drawbars are commonly offered on many forestry trailers. This last solution enables the trailer to follow tractor wheel tracks when turning and reduces the need for a wider road in switchback curves.

Even if a tractor with a forest trailer is considered a handy transportation system in forest roads with severe gradients, in the case of long logs transportation such system can be still inadequate when narrow switchback curves have to be run. In the specific scenario of Italian Alps, logs transportation along forest roads most typically makes use of a tractor and trailer system. The performance in terms of maneuverability of such a system is in general acceptable when logs are within 6 m long: on the other hand, when log length exceeds 8-10 $\mathrm{m}$, maneuverability sensibly worsens. As a consequence, forest trailers with improved steering system need to be implemented.

In order to verify the feasibility to transport logs with a length exceeding 8-10 $\mathrm{m}$ on narrow and steep forest roads, a prototype of forest double steering trailer was analyzed. The study aimed to evaluate the effectiveness of the technical solution adopted by the transportation system analyzing through a CAD simulation which considered the minimum radius of curvature and curve widening, and the maneuverability, with different length of transported logs. The results of the CAD simulation were then verified by a field test carried out on a forest road in the alpine area of Belluno province.

\section{Materials and methods}

\section{Description of the trailer prototype}

The prototype is a forest double steering trailer, based on an improved design of the so called "stinger steer truck" configuration. A sketch of the trailer is proposed in Figure 1.

It is a two axle trailer, featuring a front drive axle with differential wheels, coupled with the tractor power take-off shaft, and a twin wheeled idle axle in the rear part. The front part of the trailer is hitched to the rear frame through a central hinge giving an articulated frame. Therefore the trailer is designed to allow the idle axle to rotate relative to the hinge, and to permit also small roll and pitch motions.

The front frame protrudes rearward of the front axle wheels (the so called stinger part in stinger steer trucks), and symmetrically the rear frame protrudes frontward of the rear axle (the so called reach part). Through the hinge, the stinger steers the reach: in such a way the 
direction of back twin wheels is mechanically controlled and adapted by driving axle rotations.

Two conventional log bunks are pivotally mounted respectively on the front and on the rear frame of the trailer, and complementary support the logs. Logs constrain rear axle distance from the front one, pulling the rear part of the trailer. When the trailer is driven around a curve, orientation and distance between the bunks remain fixed due to the logs constrain, while the reach has to lengthen due to the relative rotation of the two axles. Such extension is compensated by a steel cylindrical telescoping bar implemented between the reach and the hinge on the stinger (the compensator): even when turning on roads with tight turning radii and independently of the load, such element lengthening eventually allows the relative rotation of the rear axle.

\section{Modeling}

When the tractor drives the trailer around a curve, particular attention has to be paid to two part of the vehicle: the wheels and the dynamic envelope. Indeed during motion, not only the wheels have to lie always within the roadway, but also vehicle dynamic envelope has not to interfere with elements (e.g. plants or walls) alongside the roadway.

Since the vehicle is designed to work at relatively low speeds, a kinematic slip-free condition can be assumed between inner and outer wheels, according to Ackermann condition (Jazar, 2009), getting to the following mathematical model.

With reference to Figure 2, said $\alpha_{i}$ and $\alpha_{e}$ the steer angles of respectively the inner and the outer front wheel of the tractor, and $l$ the wheelbase, the mass center of the tractor turns on a circle with radius:

$$
R_{k+1} \sqrt{a_{2}^{\frac{1}{2}} \mid J^{2} \cdot \cot ^{2} \alpha}
$$

where:

$$
\alpha \cot ^{1}\left(\frac{\cos \alpha_{1} 1 \cos \alpha_{2}}{2}\right)
$$

and the front part of the trailer follows a circular trajectory with radius $R_{T f}$ :

$$
\mathrm{R}_{\mathrm{Tr}} \sqrt{1+\cos \alpha_{1}\left|w / 2 j^{2}\right| \mathrm{b}_{1}^{2} \quad b_{2}^{2}}
$$

with a relative angle $\beta$ with respect to the tractor direction, which is expressed by:

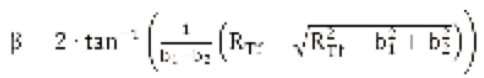

Thus the central hinge of the trail turns on a circle with radius:

$$
R_{T} \sqrt{b_{3}^{2} \mid R_{T}^{2}}
$$

The rear part of the trail, constrained by the logs length between bunks $L$, has a relative angle with respect to the front part of the trail expressed by:

$$
r \sin ^{2}\left(\frac{L}{s_{1}}\right)
$$

and the rear part of the trailer follows a circular trajectory with radius $R_{T r}$ :

$$
R_{T r} R_{T} \cdot \cos \left(\sin ^{1}\left(\frac{L}{R_{1}}\right) \quad \sin ^{1}\left(\frac{s_{1}}{\mathrm{~N}_{1}}\right)\right)
$$

Knowing the average track between the wheels of the tractor $\left(w_{M}\right)$ and of the trailer $\left(w_{T f}\right.$ and $w_{T r}$ respectively for the front and the rear axle) and the overall width of the tires $\left(t_{M}, t_{T f}\right.$ and $t_{T r}$ respectively for the tractor the front wheels and the rear wheels of the trailer), it is possible to calculate the minimum curve radius (measured in the inner side of the road) which can be run from the vehicle:

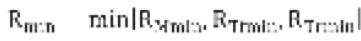

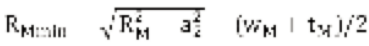

$$
\begin{aligned}
& R_{\text {Tfm:n }} \quad R_{T I} \quad\left[w_{T r} \mid t_{T r} I_{i} / 2\right.
\end{aligned}
$$

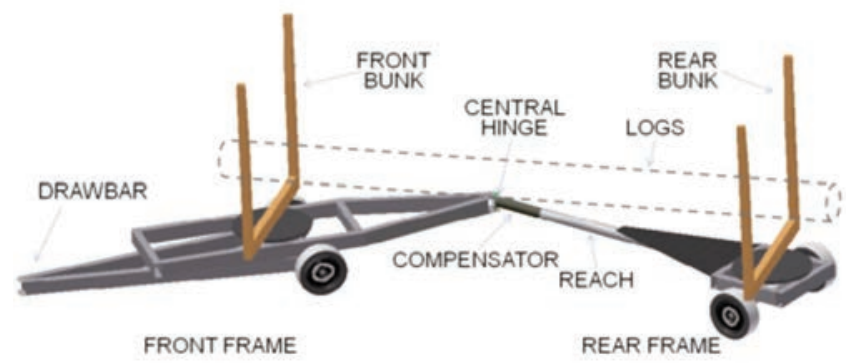

Figure 1. Schematic three-dimensional view of the trailer: a compensator implemented between a reach and a stinger allows rotation of the rear part relatively to the front part, while logs held by front and rear bunks constrain axles distance.

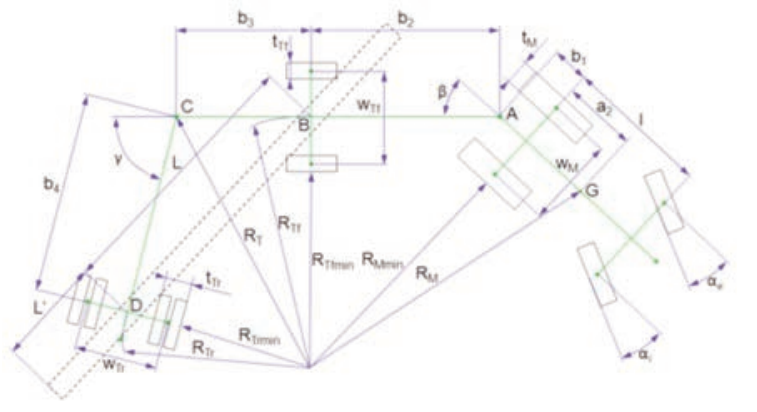

Figure 2. Simplified schematic view of the tractor with trailer and main dimensions.

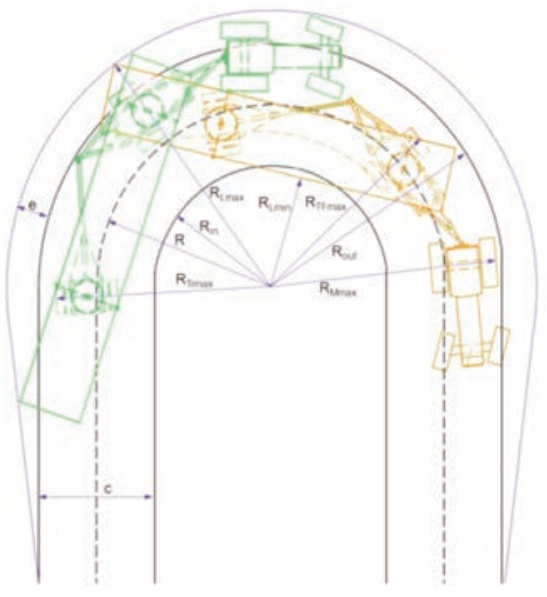

Figure 3. Schematic view of the tractor with trailer driven into a switchback curve and main dimensions. 


$$
R_{\text {Trmin }} \quad R_{T r} \quad\left[\omega_{T r} \mid E_{T r}\right] / 2
$$

The minimum curve radius $R_{\min }$ has to be compared with the inner curve radius $R_{\text {in }}$ and the following condition has to be verified: $R_{i n} \leq$ $R_{\min }$.

Similarly the maximum curve radius (measured in the outer side of the road) which can be run from the vehicle in first approximation can be calculated as:

$$
R_{\max } \max \left|R_{\text {MIm:ax }} \cdot R_{\text {T'max. }} \cdot R_{\text {T:mu: }}\right|
$$

where:

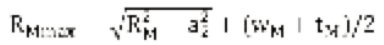

$$
\begin{aligned}
& \mathrm{R}_{\mathrm{Tfmax}} \quad \mathrm{R}_{\mathrm{Tr}} \mid\left[w_{\mathrm{Tr}} \mid \mathrm{t}_{\mathrm{Tr}}\right) / 2 \\
& R_{T r m a:} \quad R_{T r} \mid\left[w_{T r} \mid E_{T r} j / 2\right.
\end{aligned}
$$

The maximum curve radius $R_{\max }$ has to be eventually compared with the outer curve radius $R_{\text {out }}$, and the following condition has to be verified: $R_{\text {out }} \geq R_{\max }$.

Additionally the dynamic envelope of the logs and in particular minimum and maximum radius of curvature traced by the logs $R_{L m i n}$ and $R_{\text {Lmax }}$ has also to be taken into account: indeed the projection of the logs has not to exit the roadway, or at least not to interfere with elements (e.g. standing trees or walls) alongside the roadway.

\section{Software simulations}

For the present study a mechanical solid modeling design software (Autodesk Inventor ${ }^{\circledR)}$ was implemented. Such software, generally referred as 3D CAD, allows design, visualization and modeling of threedimensional digital prototypes, simulating different operating conditions in terms of loads and relative movements.

Implementation of simulation software in general brings clear advantages in design and testing of new vehicles: reduces time and costs for development of new products, allows mechanical verification of different design variations both for single elements and for whole structures and eventually allows dynamic simulation to foresee vehicle maneuverability.

For the specific study, 3D design software was implemented in order to recognize vehicle critical parts and verify their mechanical behavior when undergoing high solicitations. Additionally CAD simulations allowed foreseeing trailer dynamics, to better understand motion trajectories and maneuverability (Figure 4).

\section{Field tests}

The GPS technology applications for collecting resource measurement and managing forestry operations is well known both for off-roads (Veal et al., 2001; Mc Donald et al., 2005; Pellegrini et al., 2013) and onroads transportation (Holzleitner et al., 2011; Simwanda et al., 2011). In order to field testing the simulated forest double steering trailer, an existing prototype with a hinge position approximately at the $55 \%$ of the wheelbase length was investigated (Figure 5; Figure 6). The trailer motion was monitored through field survey using a GPS device (Trimble ${ }^{@}$ ProXH coupled with a Trimble ${ }^{@}$ NOMAD). Additionally a GIS (ArcGIS ${ }^{@} 10.1$ ) view of GPS data was implemented, allowing verifica-

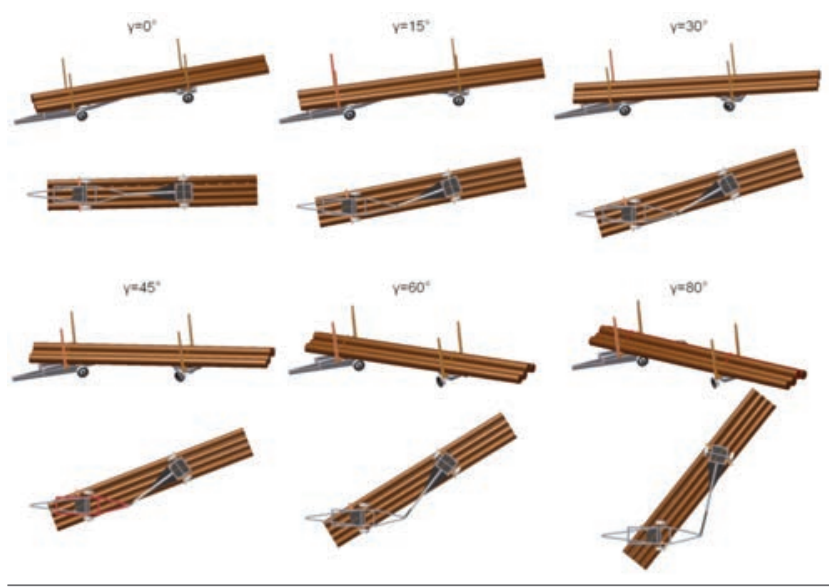

Figure 4. Simulated perspective and bottom view of the trailer at different angles $Y$ between the front and the rear part.
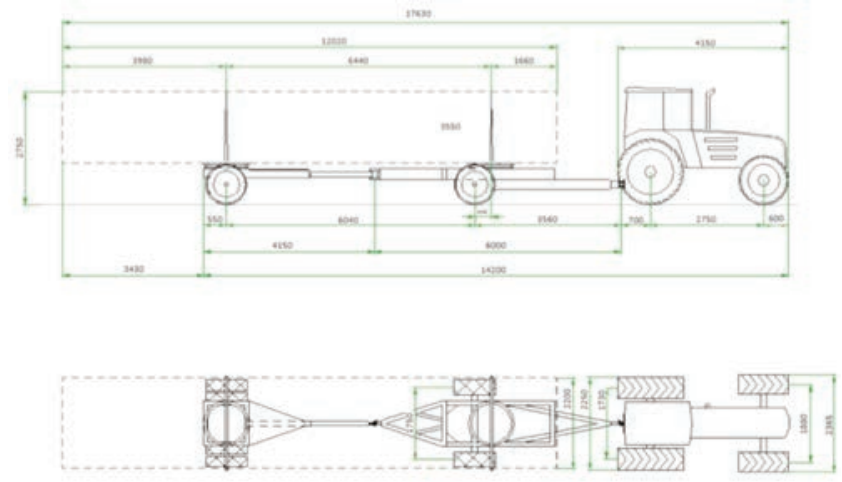

Figure 5. Layout and main dimensions $(\mathrm{mm})$ of the double steering forest trailer prototype.

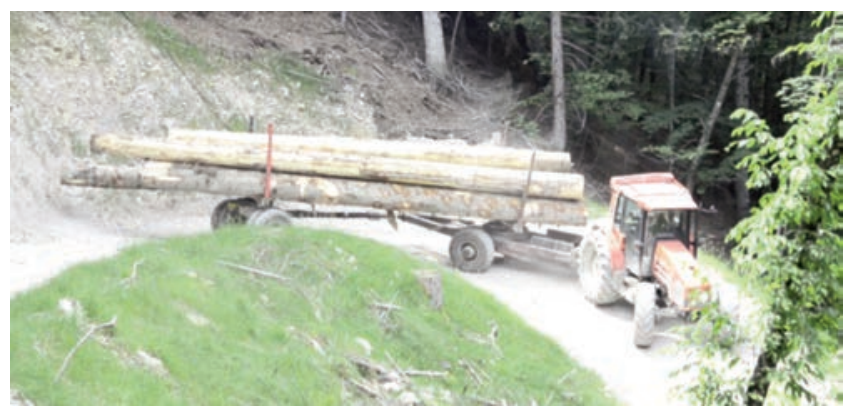

Figure 6. The double steering forest trailer prototype during the test. 
tion of the transportation movement along the forest roads, for a better understanding of trailer dynamics and maneuverability. The test considered the use of a D-GPS mounted on the front part of the farm tractor in center position above the front axle. For the analysis, the trailer was driven several times along a $6 \mathrm{~km}$ forest road in a forest site on the Eastern Italian Alps. The road showed an average width of $2.7 \mathrm{~m}$ and an average road grade of $16 \%$, with a maximum grade of $20 \%$. Switchback curves generally presented a curve radius ranging between $6 \mathrm{~m}$ and 7 $\mathrm{m}$. For the test the trailer was loaded with logs longer than $10 \mathrm{~m}$ and having an average diameter of $45 \mathrm{~cm}$. The D-GPS measurements were corrected in post-processing with the support of a public antenna available at a distance of $5 \mathrm{~km}$ from the test site.

\section{Results}

\section{Curve dynamic analysis}

The trailer underwent a set of simulations, in order to understand the role of the central hinge into curve dynamics. For this reason a twoaxle trailer with a $6 \mathrm{~m}$ wheelbase was simulated, and the effect of hinge position was varied from a minimum of zero to $100 \%$ of the wheelbase.

The minimum value $0 \%$ corresponds to a hinge positioned in correspondence of the center of the front axle: in other words corresponds to a standard two axle trailer with turntable front axle. Conversely, when the hinge is at $100 \%$ of the wheelbase, the condition corresponds to a two-axle stinger steer trailer with a stinger part as long as the wheelbase.

\section{Wheels trajectory}

Hinge position has no effect on the radius of curvature of the front axle, which is in first approximation insensitive to what stands behind. On the other hand, hinge position has a remarkable effect on trailer rear part.

In terms of radius of curvature, the closer is the hinge to the front axle, the smaller is the radius of curvature traced by the rear wheels (Figure 7). This result brings to a faulty conclusion that the closer is the hinge to the front axle the better is the maneuverability.

This conclusion is not correct: indeed for best maneuverability of the trailer it is desirable to have not just the minimum curvature, but rather the minimum off-tracking between front and rear axles of the trailer. Therefore the maximum difference between the minimum and the maximum radii $\Delta R_{T}$ measured between front and rear outer and inner wheels has to be conveniently taken into account. Such difference has a lower bound which is the track augmented by the width of the tire (i.e. the distance between outer sidewalls of inner and outer wheels):

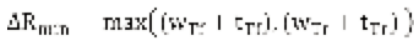

The parameter $\Delta R_{T}$ represents the roadway width needed by the trailer to complete a curve. The closer is the difference $\Delta R_{T}$ to the lower bound $\Delta R_{\text {min }}$, the smaller is the width needed to maneuver the trailer along the curve.

Simulations showed that the minimum difference is achieved when the hinge is positioned at a distance from the front axle varying between $40 \%$ and $80 \%$ of the wheelbase, with an optimum close to $60 \%$ (Figure 8). For good maneuverability of the vehicle, not only the positioning of the wheels relatively to the road site but also the dynamic envelope has to be considered, with particular attention to the hinge and the logs. The displacement of the hinge should be as small as pos-

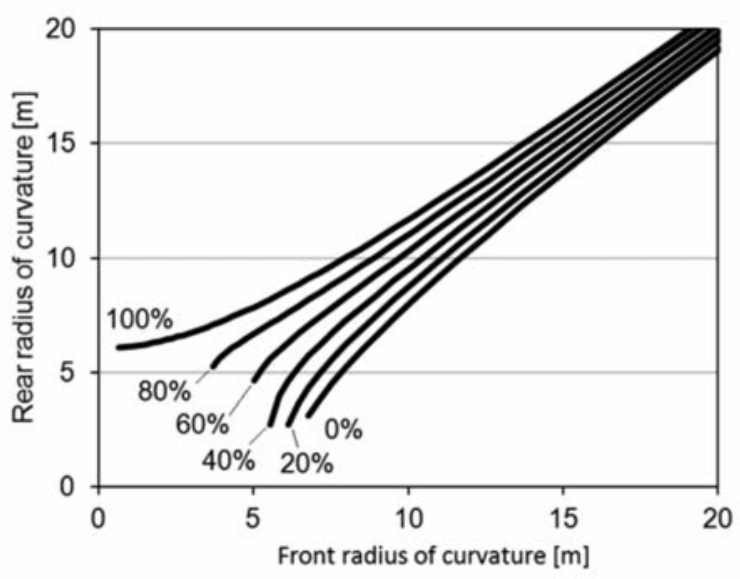

Figure 7. Variation of the rear radius of curvature RTr as a function of the front radius of curvature RTf, at different hinge positions.

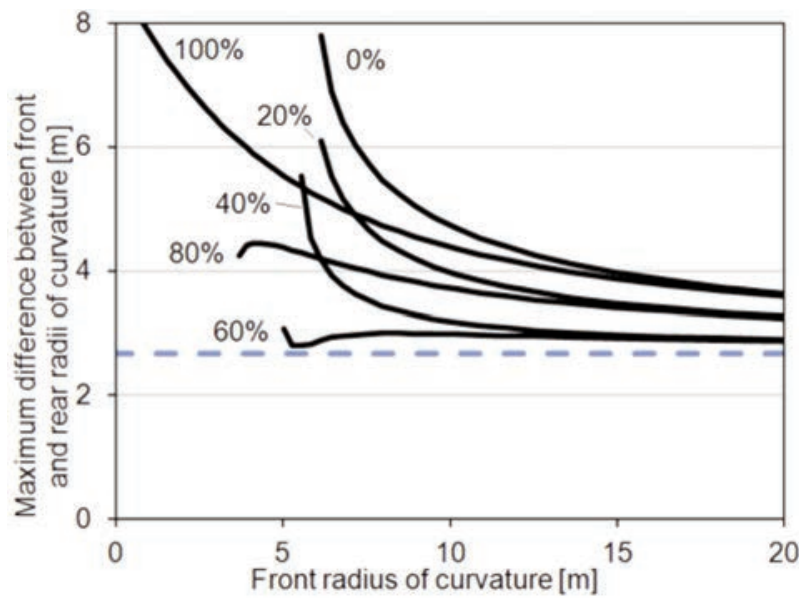

Figure 8. Variation of the difference between front and rear radii of curvature $\nabla \mathbf{R T}$ as a function of the front radius of curvature RTf; the blue dotted line represent the lower bound, given by the larger axle width.

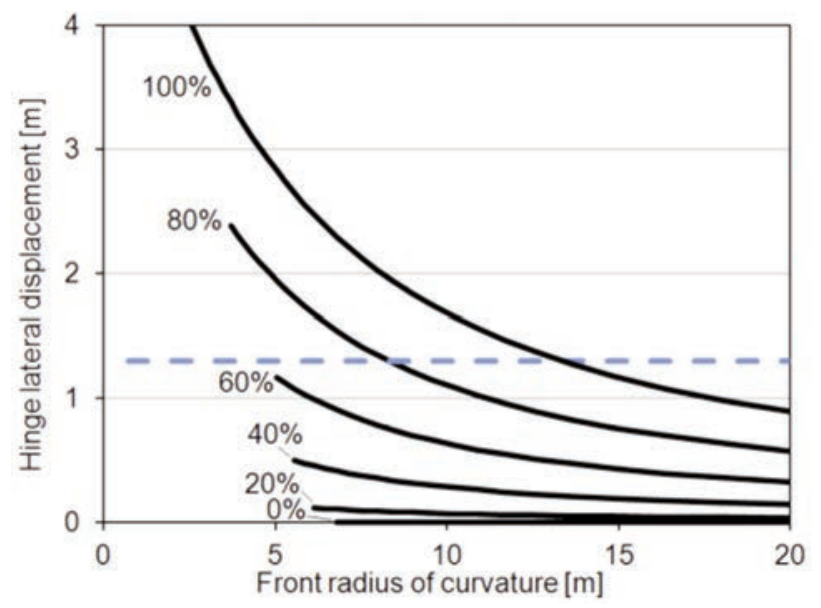

Figure 9. Displacement of the hinge as a function of the front radius of curvature RTf. 
sible: indeed even a small interference with external obstacles could bring relevant damages, causing a lock of the two part of the frame, and bringing the trailer out of order. On the other hand the horizontal projection of the logs can exit the road site: nevertheless it has to be as small as possible, in order to minimize the probability of interferences with external obstacles lying alongside the roadway.

\section{Hinge trajectory}

Then simulations were carried out, in order to evaluate central hinge displacement and logs dynamic envelope. The same two-axle trailer with $6 \mathrm{~m}$ wheelbase was considered, and again the effect of hinge position was varied from a minimum of zero to $100 \%$ of the wheelbase. Results are summarized in Figure 7, where the blue dotted line represents half the larger axle width: if the displacement overcomes such values, the hinge is supposed to sticking out from the trailer frame projection. When the hinge is at $0 \%$ of the wheelbase, the condition resembles the trivial case of a standard two-axle trailer with turntable front axle: in such state the displacement is constantly equal to zero, independently from front axle radius of curvature. As the hinge moves rearward, the displacement increases. Such displacement comes to critical values as the hinge get closer to the rear axle: in particular high displacements are found with the hinge positioned at a distance larger than $60 \%$ of the wheelbase from the front axle.

\section{Logs trajectory}

It has been said that the horizontal projection of the logs also plays an important role. Since the tighter trajectory is traced by the rear axle, analysis considered the difference between the minimum radius of curvature traced by the logs projection and the radius of curvature of the external sidewall of the rear wheel standing in the inner part of the curve. The minimum radius of the logs $R L_{\text {min }}$ was measured between the bunks in the portion of $\log$ most protruding during curve movement (see Figure 3). Results are reported in the graph below (Figure 8). In order to minimize the probability of interferences with external obstacles, the projection of the logs should be always within the wheel tracks. This occurrence is not always possible when using a two axle stinger steer trailer. In particular, as the hinge moves rearward, the probability of interferences with external obstacles increases, since the relative displacement of the logs with respect to the inner wheel $R_{\text {Lmin }}{ }^{-}$ $R_{\text {Trmin }}$ becomes negative. In other words, as the hinge gets closer to the rear axle, the logs minimum radius of curvature becomes smaller than the inner wheel radius of curvature (i.e. $R_{\text {Lmin }}<R_{\text {Trmin }}$ ). To keep the difference always positive (i.e. to avoid interferences of the central part of the logs with external obstacles), the hinge has to be kept at a distance from the front axle smaller than $40 \%$ of the wheelbase.

Analyses considered also the maximum radius of curvature produced by the dynamic envelope of the logs. In case of transportation of long logs, the larger trajectory is covered by their extremities, sticking out from the rear part of the trailer. The difference between the maximum radius of curvature traced by the logs projection and the radius of curvature of the external sidewall of the rear wheel standing in the outer part of the curve $R_{\text {Lmax }}-R_{\text {Trmax }}$ was therefore quantified (Figure 11 and 12). For the simulation $10 \mathrm{~m}$ and $12 \mathrm{~m}$ long logs were considered, with respectively a $2 \mathrm{~m}$ and a $4 \mathrm{~m}$ rear sticking out length $L^{\prime}$ (Figure 2). It is clear how tighter curvatures cause higher widening of the curve, but the effect is somehow reduced as hinge gets closer to the rear axle. The effect is clearly less evident as shorter logs are carried, on the other hand becomes more evident as their length increases.

A best configuration for a two-axle stinger steer trailer cannot be defined a priori. The design necessarily has to take into account the characteristics of the road, of the road sides and of the load type. Comparing the graphs above, in general best tradeoffs can be expected

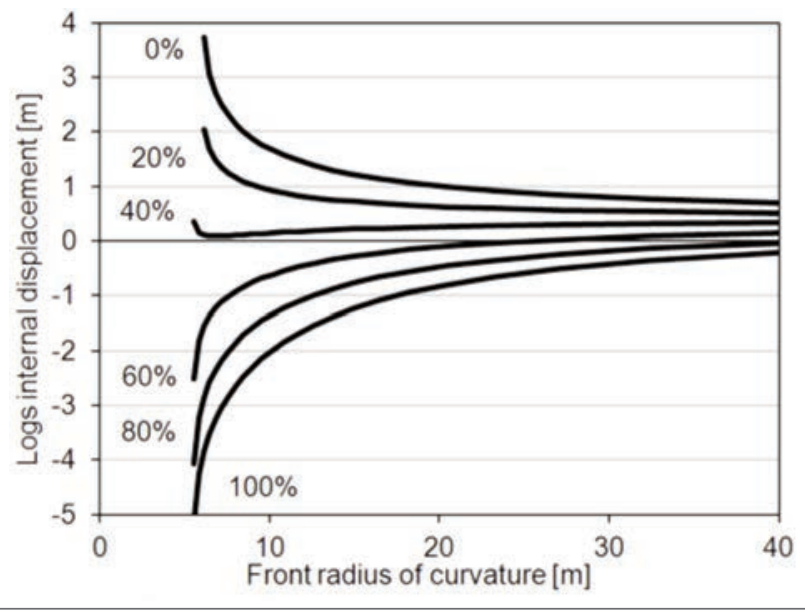

Figure 10. Logs internal displacement (measured as the difference between rear wheels and logs minimum radius of curvature $R_{L \min }-R_{T r m i n}$ ) as a function of the front radius of curvature.

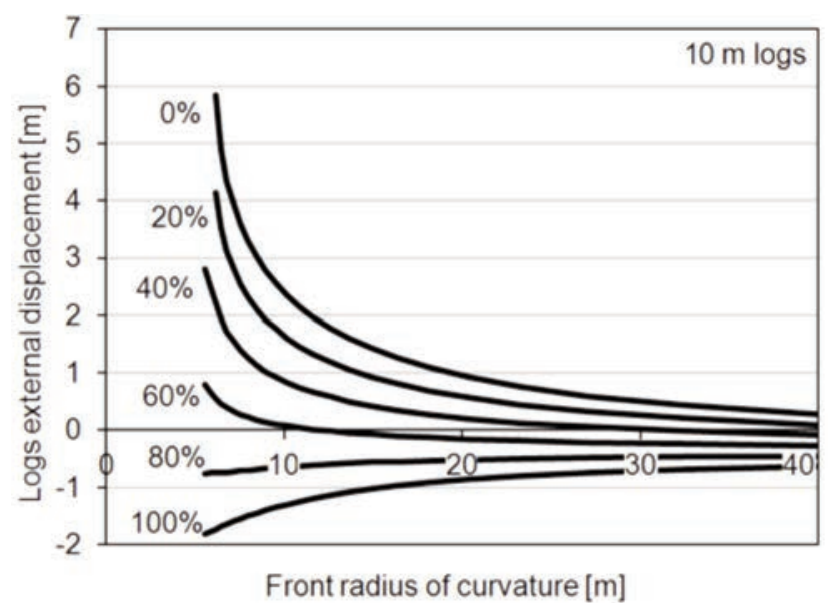

Figure 11. Logs external displacement (measured as the difference between rear wheels and logs maximum radius of curvature $R_{L \max }-R_{T r m a x}$ ) as a function of the front radius of curvature in the case of $10 \mathrm{~m}$ logs.

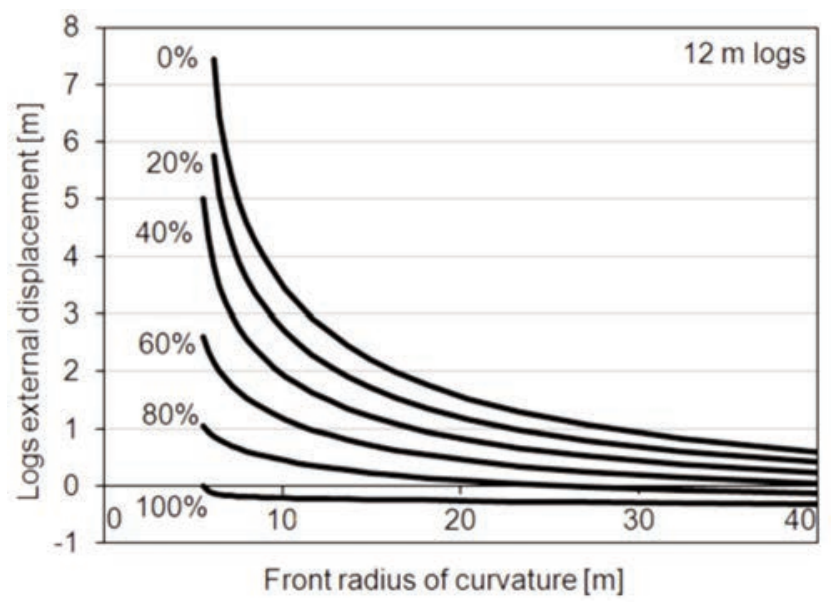

Figure 12. Logs internal displacement (measured as the difference between rear wheels and logs maximum radius of curvature $R_{L \max }-R_{\text {Trmax }}$ ) as a function of the front radius of curvature in the case of $12 \mathrm{~m}$ logs. 
whenever the hinge is positioned at a distance from the front axle ranging between $40 \%$ and $60 \%$ of the wheelbase, but unfortunately a general statement is not possible.

\section{Field tests}

The test carried on the double steering forest trailer prototype highlights a good maneuverability of the transportation system along forest road with high road grade and small switchback curves radius. Both the conditions with loaded and unloaded trailer were tested.

In the case of loaded trailer with logs longer than $10 \mathrm{~m}$ (the longest with a length of $12 \mathrm{~m}$, positioned at the center of the trailer), the transportation system was clearly affected by the high transported payload (average speed in loaded condition $4.9 \mathrm{~km} \mathrm{~h}^{-1}$, average speed in unloaded condition $9.5 \mathrm{~km} \mathrm{~h}^{-1}$ ). The minimum switchback curve radius was $6 \mathrm{~m}$ with an external widening curve of approximately of $1 \mathrm{~m}$. Such result confirmed simulations, which foresaw an external logs displacement of about $1.5 \mathrm{~m}$ (see Figure 9).

The back projection of the logs (approximately $3 \mathrm{~m}$ ) played an important role during the approach of the switchback curve as the projection of the longest log was very close to the cut-slope of the road prism. For this reason the tractor driver had to continuously modify and correct tractor trajectory acting on the front wheel direction. The result is a trajectory where the center of curvature is not a fixed point coincident with the curve center: conversely the center of curvature of the vehicle is constantly changing during motion. On the other hand, also the curve has not a constant curvature but the radius constantly decreases in the first half, and symmetrically increases during the second half. This behavior is typical of all the roads, and introduces a limitation in reported simulations. Indeed proposed analyses are built on the condition of constant radii of curvature, which is not verified in actual roads. Nevertheless the relevance of the proposed results is not diminished, posing the limits for the maximum performance achievable with the trailer driven in ideal conditions.

\section{Conclusions}

In this study a prototype of a double steering forest trailer for long log transportation was analyzed and tested. The simulation of the prototype was supported by dedicated 3D software mainly focusing on the wheels and the dynamic envelope when the transportation system runs switchback curves with challenging curve radii.

The simulation results evidenced how the hinge position influences the radius of curvature of the rear wheels. The simulation allowed identifying an optimum position (between 40 to $60 \%$ of the wheelbase respect to the front axle) of the hinge to minimize the off-tracking between front and the rear axle. Such hinge positioning is also suitable to avoid excessive lateral displacement of the hinge, keeping its trajectory always within the track of the trailer.

Simulations evidenced also how logs external displacement is minimized when the hinge is brought closer to the rear axle, however stay- ing within acceptable values when it is positioned at least at $50 \%$ of the wheelbase length.

Tests on a prototype (with the hinge location approximately at $55 \%$ of the wheelbase) reasonably confirm simulation results in term of transportation system dynamic. Main limitations arise from forest roads poor regularity, which typically replicates the complex morphology of the mountainous terrain. In such conditions software simulations are not sufficient to accurately describe the dynamic of the transportation system, but gives however relevant indications on maximum expectable performance.

\section{References}

Baldini S., Cavalli R., Piegai F, Spinelli R., Di Fulvio F., Fabiano F., Grigolato S., Laudati G., Magagnotti N., Nati C., Picchio R. 2009. Prospettive di evoluzione nel settore delle utilizzazioni forestali e dell'approvvigionamento del legname. Atti del Terzo Congresso Nazionale di Selvicoltura per il miglioramento e la conservazione dei boschi italiani. Taormina, 16-19 0ttobre 2008, Firenze: Accademia Italiana di Scienze Forestali, 2:717-728, ISBN/ISSN: 978-88-87553-16-1.

Bont L.G., Heinimann H.R., Church R.L. 2012. Concurrent optimization of harvesting and road network layouts under steep terrain. Ann Oper Res. 1-24.

Cavalli R., Grigolato S. 2010. Influence of characteristics and extension of a forest road network on the supply cost of forest woodchips. J For Res-JPN. 15:202-209.

Holzleitner F., Kanzian C., Stampfer K. 2011. Analyzing time and fuel consumption in road transport of round wood with an onboard fleet manager. Eur J For Res. 130:293-301.

Jazar R.N. 2009. Mathematical theory of autodriver for autonomous vehicles. J Vib Control. 16: 253.

Jones D.H. 1995. Timber trailers for agricultural tractors. Forest Research. Technical Notes. 28. Machynlleth: Forest Commission.

McDonald, T. P., \& Fulton, J. P. (2005). Automated time study of skidders using global positioning system data. Comput Electron Agr. 48(1):19-37.

Pellegrini M., Ackerman P., Cavalli R. 2013. On-board computing in forest machinery as a tool to improve skidding operations in South African softwood sawtimber operations. South Forests. 75:89-96.

Sessions J., Wimer J., Boston K. 2009. Increasing Value and Reducing Costs through Hauling Longer Logs: Opportunities and Issues. West J Appl For. 24:157-162.

Simwanda M., Wing M.G., Sessions J. 2011. Evaluating global positioning system accuracy for forest biomass transportation tracking within varying forest canopy. West J Appl For. 26:165-173.

Stückelberger J.A., Heinimann H.R., Burlet E.C. 2006. Modeling spatial variability in the life-cycle costs of low-volume forest roads. Eur J For Res. 125:377-390.

Veal M.W., Taylor S.E., McDonald T.P., McLemore D.K., Dunn M.R. 2001. Accuracy of tracking forest machines with GPS. T ASAE. 44:19031911. 